\title{
A Conventional New Procedure for $N$-Acylation of Unprotected Amino Acids
}

\author{
Fumiko FujISAKi, Marumi Oishi, and Kunihiro Sumoto* \\ Faculty of Pharmaceutical Sciences, Fukuoka University; Nanakuma, Jonan-ku, Fukuoka 814-0180, Japan. \\ Received August 8, 2006; accepted September 5, 2006
}

The preparation of amide derivatives (4) by $N$-acylation of unprotected $\alpha$-amino acids is easily achieved via readily available benzotriazolyl carboxylates $(2 \mathrm{a}-\mathrm{d})$ or succinimidyl carboxylates $(2 \mathrm{e}-\mathrm{f})$. These intermediates (2) are prepared from reaction of carboxylic acids (1) with 1-hydroxybenzotriazole (HO-Bt) or $N$-hydroxysuccinimide (HO-Su) in the presence of equimolar amounts of 1-ethyl-3-(3'-dimethylaminopropyl)carbodiimide hydrochloride (WSCI). The overall yields of the target compounds (4) were excellent, and this two-stage procedure could be applicable as an alternative procedure for one-pot reaction.

Key words unprotected amino acid; $\beta$-aminoalanine; $N$-acylation; 1 -hydroxybenzotriazole; $N$-hydroxysuccinimide; 1 -ethyl-3(3'-dimethylaminopropyl)carbodiimide

Preparation of $\mathrm{N}$-acyl derivatives of amino acids from acyl halides (usually chlorides) and amino acids is well known in organic synthesis, the so-called Schotten-Baumann reaction. $^{1)}$ Many of the coupling reagents for the formation of amide bonds in the synthesis of biologically active compounds have been reported. ${ }^{2)}$ Recently, a few effective coupling reagents for $\mathrm{N}$-acylation of unprotected amino acids have been reported. ${ }^{3-7)}$ For instance, the coupling reaction utilizing $N$-acyl benzotriazole derivatives and unprotected amino acids proceeds under mild reaction conditions. ${ }^{7)}$

Recently, we reported that $N$-acyl derivatives of $\beta$ aminoalanines were synthesized under Schotten-Baumann reaction conditions with acyl halides. ${ }^{8,9)}$ For further structural modification of $N$-acyl derivatives of $\beta$-aminoalanines in the search for biologically active compounds, we were in need of a conventional method for the synthesis of some $N$-acyl $\beta$ aminoalanines. In the course of our studies, we found that the combination of 1-ethyl-3-(3'-dimethylaminopropyl)carbodiimide hydrochloride (WSCI) ${ }^{3,10)}$ and 1-hydroxybenzotriazole $(\mathrm{HO}-\mathrm{Bt})^{3)}$ or 1-hydroxysuccinimide (HO-Su $)^{3)}$ for such coupling reactions is effective to obtain the $N$-acylated derivatives from unprotected $\beta$-aminoalanines. We report herein a simple conventional method for the $\mathrm{N}$-acylation of unprotected amino acids.

\section{Results and Discussion}

As shown in Tables 1 and 2, the use of equimolar amounts of $\mathrm{WSCI}^{10)}$ gave almost quantitative yields of the desired ac- tivated carboxylic acid (2), and this procedure may be efficiently exploited in the case of starting acids with an amine functionality [see entries 2, 3 (Table 1) and entries 2, 3, and 5 (Table 2), respectively].

The structures of the activated derivatives $(\mathbf{2 a}-\mathbf{d})$ were easily confirmed from the spectroscopic and elemental analysis. Thus IR spectra of compounds $\mathbf{2 a}-\mathbf{d}$ showed a characteristic absorption band at $1809-1781 \mathrm{~cm}^{-1}$ ascribable to the $\mathrm{C}=\mathrm{O}$ group in the molecules. In the ${ }^{1} \mathrm{H}-\mathrm{NMR}$ spectra, four aromatic protons of benzotriazole ring were observed at $\delta 7.26-8.14 \mathrm{ppm}$ as multiplets. Regarding the benzotriazole ring in compounds $\mathbf{2 a}-\mathbf{d}$, six ${ }^{13} \mathrm{C}$-signals were observed at $\delta$ $108.3-143.6 \mathrm{ppm}$ elucidating the representative structure (2).

Other assignments of NMR data of the products are given in Experimental. In the case of derivatives $(\mathbf{2} \mathbf{e}-\mathbf{f})$, IR spectra showed three absorption bands at $1821-1730 \mathrm{~cm}^{-1}$, ascribable to the two $\mathrm{C}=\mathrm{O}$ groups in the succinimide ring and a $\mathrm{C}=\mathrm{O}$ for $-\mathrm{COON}=$ groups. Other physical data (FAB-MS and elemental analysis) were in good agreement with the structures (2) (see Experimental). These assignments and correlation of ${ }^{1} \mathrm{H}$ - and ${ }^{13} \mathrm{C}$-NMR signals were supported by 2D spectroscopic analysis.

Table 2 shows the results of reactions of these activated carboxylic acid derivatives (2) with unprotected amino acids (3). The structures were confirmed by spectroscopic and physical data of the target amide derivatives (4). Thus IR spectra $(\mathrm{KBr})$ showed two typical absorption bands at

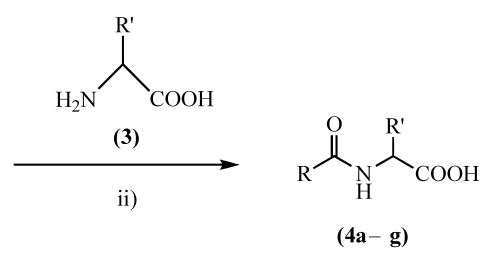

$$
\begin{array}{lc}
\mathrm{Bt}=\text { benzotriazol-1-yl } & \mathbf{2 a}-\mathbf{d}: \mathbf{B t} \\
\mathrm{Su}=\text { succinimid-1-yl } & \mathbf{2 e}-\mathbf{f}: \mathbf{S u} \\
\text { WSCI = 1-ethyl-3-(3'-dimethylaminopropyl)carbodiimide hydrochloride } & \\
& \\
\text { i) } \mathrm{Bt}-\mathrm{OH} \text { (or } \mathrm{Su}-\mathrm{OH}), \text { WSCI; } & \text { ii) aqueous } \mathrm{K}_{2} \mathrm{CO}_{3}
\end{array}
$$


Table 1. Preparation of Activated Carboxylic Acids

\begin{tabular}{|c|c|c|c|c|c|c|c|c|c|}
\hline Entry & \multicolumn{2}{|c|}{$\mathrm{R}-\mathrm{COOH}(\mathbf{1})$} & \multirow{2}{*}{$\begin{array}{c}\text { Additive } \\
\text { HO-Bt }\end{array}$} & \multirow{2}{*}{$\begin{array}{l}\text { Solvent } \\
\mathrm{CH}_{2} \mathrm{Cl}_{2}\end{array}$} & \multirow{2}{*}{$\begin{array}{c}t_{\mathrm{R}} \\
40 \mathrm{~min}\end{array}$} & \multicolumn{2}{|c|}{$\begin{array}{c}\text { Activated } \\
\text { carboxylic acid (2) }\end{array}$} & \multirow{2}{*}{$\begin{array}{c}\text { Yield }(\%)^{a)} \\
98\end{array}$} & \multirow{2}{*}{$\begin{array}{c}\begin{array}{c}\mathrm{mp}{ }^{\circ} \mathrm{C} \\
\text { (Solvent) }\end{array} \\
118-124 \\
\text { (AcOEt-hexane) }\end{array}$} \\
\hline 1 & $\underbrace{\mathrm{H}}_{\overline{\overline{\mathrm{H}}}} \mathrm{COPh}$ & 1a & & & & $\begin{array}{l}\mathrm{COOBt} \\
\mathrm{COPh}\end{array}$ & $2 a$ & & \\
\hline 2 & $\underset{\mathrm{F}}{\mathrm{C}} \underset{1}{\longrightarrow}\rangle-\mathrm{COOH}$ & $1 \mathrm{~b}$ & $\mathrm{HO}-\mathrm{Bt}$ & $\mathrm{DMF}$ & $10 \mathrm{~min}$ & $\underset{\mathrm{F}}{-\mathrm{COOBt}}$ & $2 b$ & 97 & $\begin{array}{l}102-103 \\
\left.\text { (iso- } \mathrm{Pr}_{2} \mathrm{O}\right)\end{array}$ \\
\hline 3 & ${ }_{\mathrm{OCH}_{3}}$ & $1 \mathrm{c}$ & $\mathrm{HO}-\mathrm{Bt}$ & DMF & $3 \mathrm{~h}$ & - $\mathrm{OCOOBt}_{3}$ & $2 c$ & 99 & $\begin{array}{l}169-170(\mathrm{dec} .) \\
\left(\mathrm{CH}_{3} \mathrm{CN}\right)\end{array}$ \\
\hline 4 & $\|-\mathrm{COOH}$ & 1d & $\mathrm{HO}-\mathrm{Bt}$ & $\mathrm{CH}_{2} \mathrm{Cl}_{2}$ & $2 \mathrm{~min}$ & $-\mathrm{COOBt}$ & 2d & 97 & $\begin{array}{l}84-85(\mathrm{dec} .) \\
\left(\text { iso- }-\mathrm{Pr}_{2} \mathrm{O}\right)\end{array}$ \\
\hline 5 & $\mathrm{COOH}$ & 1d & $\mathrm{HO}-\mathrm{Su}$ & DMF & $10 \mathrm{~min}$ & $-\operatorname{coOSu}$ & $2 e$ & 97 & $\begin{array}{l}136-138(\mathrm{dec} .)^{b)} \\
\left(\text { AcOEt-iso- } \mathrm{Pr}_{2} \mathrm{O}\right)\end{array}$ \\
\hline 6 & $\mathrm{Cl}-\mathrm{CH}_{2} \mathrm{COOH}$ & $1 e$ & $\mathrm{HO}-\mathrm{Su}$ & $\mathrm{CH}_{2} \mathrm{Cl}_{2}$ & $20 \mathrm{~min}$ & 1) $\mathrm{CH}_{2} \mathrm{COOSu}$ & $2 f$ & 99 & $\begin{array}{l}141-142 \text { (dec.) } \\
\text { (iso-PrOH) }\end{array}$ \\
\hline
\end{tabular}

a) The isolated products showed one spot by TLC analysis. b) Lit. ${ }^{14)} \mathrm{mp} 134-136^{\circ} \mathrm{C}$

Table 2. Preparation of $N$-Acylamino Acids from Compounds (2)

\begin{tabular}{|c|c|c|c|c|c|c|c|c|c|}
\hline Entry & $\begin{array}{l}\text { Compound } \\
\text { (2) }\end{array}$ & \multicolumn{2}{|c|}{ Amino acid (3) } & Solvent & $t_{\mathrm{R}}$ & \multicolumn{2}{|l|}{ Product (4) } & Yield $(\%)^{a)}$ & $\begin{array}{c}\mathrm{mp}{ }^{\circ} \mathrm{C} \\
\text { (Solvent) }\end{array}$ \\
\hline 1 & $2 a$ & $\begin{array}{c}\mathrm{COOH} \\
1 \\
\mathrm{NH}_{2} \mathrm{CHCH}_{2} \mathrm{~N} \\
{ }_{2 \mathrm{HCl}}\end{array}$ & (3a) & $\mathrm{CH}_{2} \mathrm{Cl}_{2} / \mathrm{EtOH}$ & $5 \mathrm{~h}$ & & (4a) & $89^{b)}$ & 151 (dec.) \\
\hline 2 & $2 b$ & $3 \mathbf{a}$ & & $\mathrm{CH}_{3} \mathrm{CN}$ & $0.5 \mathrm{~h}$ & & (4b) & $78^{b)}$ & 149 (dec.) \\
\hline 3 & $2 c$ & $\mathbf{3 a}$ & & $\mathrm{CH}_{2} \mathrm{Cl}_{2}$ & $1 \mathrm{~d}$ & $\begin{array}{c}\mathrm{COOH} \\
\qquad-\mathrm{CONHCHCH}_{2} \mathrm{~N} \\
\mathrm{OCH}_{3}\end{array}$ & (4c) & $86^{b)}$ & 157 (dec.) \\
\hline 4 & $2 d$ & 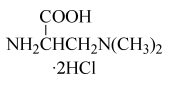 & $(3 b)$ & $\mathrm{CH}_{2} \mathrm{Cl}_{2}$ & $5 \mathrm{~h}$ & 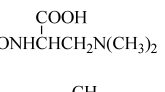 & (4d) & $76^{b)}$ & $\begin{array}{l}186(\text { dec. }) \\
(\mathrm{EtOH})\end{array}$ \\
\hline 5 & $2 c$ & Ala & & DMF & $10 \mathrm{~min}$ & ${ }_{\mathrm{OCH}_{3}}$ & $(4 e)$ & 90 & $\begin{array}{l}236 \text { (dec.) } \\
\left(\mathrm{CH}_{3} \mathrm{CN}\right)\end{array}$ \\
\hline 6 & $2 e$ & Phe & & $\mathrm{CH}_{2} \mathrm{Cl}_{2}$ & $2 \mathrm{~h}$ & $\begin{array}{c}\mathrm{CH}_{2} \mathrm{Ph} \\
\mathrm{NHCHCOOH}\end{array}$ & (4f) & 97 & $\begin{array}{l}184-185^{c)} \\
\left(\mathrm{CH}_{3} \mathrm{COOH}\right)\end{array}$ \\
\hline 7 & $2 f$ & Ala & & DMF & $10 \mathrm{~min}$ & $\begin{array}{c}\mathrm{CH}_{3} \\
\mathrm{CH}_{2} \mathrm{CONHCHCOOH}\end{array}$ & (4g) & 89 & $\begin{array}{l}169-171 \\
\left(\mathrm{CH}_{3} \mathrm{CN}\right)\end{array}$ \\
\hline
\end{tabular}

a) The isolated products showed one spot by TLC analysis. b) Purified by column chromatography $\left.\left(\mathrm{SiO}_{2}\right) . \quad c\right)$ Lit. ${ }^{1)} \mathrm{mp} 184-185{ }^{\circ} \mathrm{C}$.

$1686-1613$ and $3292-3194 \mathrm{~cm}^{-1}$ attributable to the amide $\mathrm{C}=\mathrm{O}$ and amide $-\mathrm{NH}-$ groups, respectively. In addition to these observations, compounds $\mathbf{4 a}, \mathbf{4 c}$, and $\mathbf{4 d}$ showed at $c a$. $1600 \mathrm{~cm}^{-1}$ ascribable to a carboxylate $\left(-\mathrm{COO}^{-}\right)$group, which apparently indicates that these compounds require the twitter ion structure in the solid state. ${ }^{8)}$ In all ${ }^{1} \mathrm{H}-\mathrm{NMR}$ spectra, a characteristic $\mathrm{C} \alpha$-H proton for $\alpha$-amino acids could be observed at $\delta 4.20-5.10 \mathrm{ppm}$. Other protons were also in good agreement with the represented structures of 4 . In the ${ }^{13} \mathrm{C}$-NMR spectra of the products 4 , three ${ }^{13} \mathrm{C}$ signals were observed at $\delta 163.0-175.5,171.4-176.8$, and 47.6$54.4 \mathrm{ppm}$, ascribable to a $-\mathrm{CON}=$ amide, $\mathrm{a}-\mathrm{COOH}$, and a $\mathrm{C} \alpha$ carbon, respectively. Full assignments of these data of structures 4 are recorded in Experimental. Other physical data (FAB-MS and elemental analysis) of these compounds are also in good agreement with the representative structure (4).

In our experimental results, some of the advantages of combination of $\mathrm{HO}-\mathrm{Bt}$ (or $\mathrm{HO}-\mathrm{Su}$ ) and WSCI are that the procedure by two-stage coupling reaction is quite conventional and gave isolable stable activated derivatives in excellent yields, and can effect rapid coupling. Coupling reactions with the reported reagents are frequently moisture sensitive, and include some difficulties of isolation and purification processes due to the formation of by-products from the coupling reagents. ${ }^{3,7)}$

Preparation of the coupling products by the above procedure gave excellent results in terms of the yield or easy preparation procedure of overall process. In addition, this two-stage procedure is accessible for a new procedure for one-pot amide formation based on activation of carboxylic acids in situ. For example, the preparation of $\mathbf{4 f}$ by one-pot reaction starting with $\mathbf{1 d}$ using either $\mathrm{HO}-\mathrm{Bt}$ or $\mathrm{Su}-\mathrm{OH}$ can be achieved in excellent yield (see Method A and B in Experimental). ${ }^{11)}$ The molecular modification by this conventional procedure has a wide range of applications for $\mathrm{N}$-acylation of unprotected amino acids. Further synthetic and biological studies on related compounds are in progress. 


\section{Experimental}

Melting points are uncorrected. IR spectra were measured by Shimadzu FTIR-8100 spectrometer. ${ }^{1} \mathrm{H}$ - and ${ }^{13} \mathrm{C}$-NMR spectra were obtained on a JEOL JNM A-500 $\left(500 \mathrm{MHz}\right.$ for ${ }^{1} \mathrm{H}, 125 \mathrm{MHz}$ for $\left.{ }^{13} \mathrm{C}\right)$ at $35^{\circ} \mathrm{C}$ unless otherwise noted. Chemical shifts are expressed as $\delta$ ppm downfield from an internal tetramethylsilane (TMS). Signal assignments were confirmed by ${ }^{1} \mathrm{H}-$ ${ }^{1} \mathrm{H}$ correlation spectroscopy (COSY), ${ }^{1} \mathrm{H}-{ }^{13} \mathrm{C}$ heteronuclear multiple-quantum coherence (HMQC), or ${ }^{1} \mathrm{H}-{ }^{13} \mathrm{C}$ heteronuclear multiple-bond connectivity (HMBC) spectra. FAB-MS were obtained by JEOL JMS-HX110 mass spectrometer. The preparation of racemic $\beta$-aminoalanines (3a, b) as starting materials has already been described in our previous paper. ${ }^{12)}$ The following abbreviations in brackets were used: cyclohexane ring (Cyhx), piperidine ring $(\mathrm{Ppd})$, pyrrolidine ring (Pyr), and benzotriazole group $(\mathrm{Bt})$.

Isolation of Activated Carboxylic Acid (2). General Procedure At room temperature, 1-ethyl-3-(3'-dimethylaminopropyl)carbodiimide hydrochloride (WSCI) $(5.5 \mathrm{mmol})$ was added to a solution of carboxylic acid (1) $(5.0 \mathrm{mmol})$ and 1-hydroxybenzotriazole monohydrate (HO-Bt) $(5.5 \mathrm{mmol})$ or 1-hydroxysuccinimide $(\mathrm{HO}-\mathrm{Su})(5.5 \mathrm{mmol})$ in dichloromethane $\left(\mathrm{CH}_{2} \mathrm{Cl}_{2}\right)$ or dimethylformamide (DMF) and the resulting mixture was stirred until the starting material disappeared. In case of entries 1, 4, and 6 (Table 1), the reaction mixture was washed with water. After drying over $\mathrm{Na}_{2} \mathrm{SO}_{4}$, evaporation of the solvent gave the desired products $(\mathbf{2} \mathbf{a}, \mathbf{d}, \mathbf{f})$ in almost quantitative yields. In the case of entries 2, 3, and 5 (Table 1), after addition of water the precipitates were collected by filtration or the resulting mixture was extracted with diethyl ether, washed with brine, and dried over $\mathrm{Na}_{2} \mathrm{SO}_{4}$. Evaporation of the solvent afforded good purity of the compounds $(\mathbf{2} \mathbf{b}, \mathbf{c}, \mathbf{e})$. The products could be used for the next stage without further purification.

$1 \mathrm{H}$-Benzotriazol-1-yl trans-2-Benzoylcyclohexanecarboxylate (2a) FAB-MS (positive) $m / z: 350(\mathrm{M}+\mathrm{H})^{+}$. IR $(\mathrm{KBr}) \mathrm{cm}^{-1}: 1802,1678 .{ }^{1} \mathrm{H}-$ NMR $\left(\mathrm{CDCl}_{3}\right) \delta: 1.56-1.60(3 \mathrm{H}, \mathrm{m}, \mathrm{Cyhx} \mathrm{H}-3, \mathrm{H}-4, \mathrm{H}-5), 1.78-1.84$ $(1 \mathrm{H}, \mathrm{m}$, Cyhx H-6), 1.94-1.99 (1H, m, Cyhx H-4), 2.08-2.14 (1H, m, Cyhx H-5), 2.19-2.25 (1H, m, Cyhx H-3), 2.37-2.44 (1H, m, Cyhx H-6), $3.26(1 \mathrm{H}, \mathrm{dd}, J=11.0,4.5 \mathrm{~Hz}$, Cyhx H-1), 3.94-3.98 (1H, m, Cyhx H-2), 7.26-7.41 (1H, m, Bt Ar-H), 7.47-7.60 (4H, m, Bt Ar-H $\times 2$ and $m-\mathrm{Ar}-\mathrm{H})$, $7.85(1 \mathrm{H}, \mathrm{d}, J=8.0 \mathrm{~Hz}, p-\mathrm{Ar}-\mathrm{H}), 7.93(2 \mathrm{H}, \mathrm{dd}, J=8.5,1.0 \mathrm{~Hz}, o-\mathrm{Ar}-\mathrm{H}), 8.02$ $(1 \mathrm{H}, \mathrm{d}, J=8.0 \mathrm{~Hz}, \mathrm{Bt} \mathrm{Ar}-\mathrm{H}) .{ }^{13} \mathrm{C}-\mathrm{NMR}\left(\mathrm{CDCl}_{3}\right) \delta: 25.3,25.5$ (Cyhx C-4, C5), 29.1 (Cyhx C-6), 30.0 (Cyhx C-3), 42.4 (Cyhx C-1), 47.9 (Cyhx C-2), 108.8 (Bt Ar-C), 120.2 (Bt Ar-C), 124.7 (Bt Ar-C), 128.4, 128.6, 128.7 (o, $m$-Ar-C and Bt Ar-C), 133.4 (p-Ar-C), 135.5 (Bt Ar-C), 135.7 (Ar C-1), 143.4 (Bt Ar-C), 171.7 (-COO), 202.1 (OPP). Anal. Calcd for $\mathrm{C}_{20} \mathrm{H}_{19} \mathrm{~N}_{3} \mathrm{O}_{3}$ : C, 68.75; H, 5.48; N, 12.03. Found: C, 68.72; H, 5.53; N, 12.03 .

1H-Benzotriazol-1-yl 4-(1-Piperidinyl)-3-fluorobenzoate (2b) FABMS (positive) $\mathrm{m} / z: 341(\mathrm{M}+\mathrm{H})^{+}$. IR $(\mathrm{KBr}) \mathrm{cm}^{-1}: 1782,1611 .{ }^{1} \mathrm{H}-\mathrm{NMR}$ $\left(\mathrm{CDCl}_{3}\right) \delta: 1.66-1.69(2 \mathrm{H}, \mathrm{m}, \mathrm{Ppd} \mathrm{H}-4), 1.73-1.78(4 \mathrm{H}, \mathrm{m}, \mathrm{Ppd} \mathrm{H}-3, \mathrm{H}-$ 5), 3.30-3.33 (4H, m, Ppd H-2, H-6), $7.00(1 \mathrm{H}, \mathrm{t}, J=8.5 \mathrm{~Hz}, \mathrm{Ar} \mathrm{H}-2)$, 7.27-7.45 (2H, m, Bt Ar-H), 7.52-7.55 (1H, m, Bt Ar-H), $7.84(1 \mathrm{H}, \mathrm{dd}$, $J=4.0,2.0 \mathrm{~Hz}, \mathrm{Ar} \mathrm{H}-5), 7.94-7.97(1 \mathrm{H}, \mathrm{m}, \mathrm{Ar} \mathrm{H}-6), 8.08(1 \mathrm{H}, \mathrm{d}, J=8.0 \mathrm{~Hz}$, Bt Ar-H). ${ }^{13} \mathrm{C}-\mathrm{NMR}\left(\mathrm{CDCl}_{3}\right) \delta: 24.2$ (Ppd C-4), 25.8 (Ppd C-3, C-5), 50.9 (Ppd C-2 , C-6), 108.4 (Bt Ar-C), 114.8 (Ar C-1), 117.9 (Ar C-2, J=4.0 Hz), 118.4 (Ar C-5, $J=24.0 \mathrm{~Hz}$ ), 120.5 (Bt Ar-C), 124.7 (Bt Ar-C), 128.2 (Ar C6), 128.6 (Bt Ar-C), 129.0 (Bt Ar-C), 143.6 (Bt Ar-C), 146.9 (Ar C-4, $J=7.0 \mathrm{~Hz}), 153.6(\mathrm{Ar} \mathrm{C}-3, J=247.0 \mathrm{~Hz}), 161.9(\underline{\mathrm{CO}})$. Anal. Calcd for $\mathrm{C}_{18} \mathrm{H}_{17} \mathrm{~N}_{4} \mathrm{O}_{2} \mathrm{~F}: \mathrm{C}, 63.52 ; \mathrm{H}, 5.03 ; \mathrm{N}, 16.46$. Found: $\mathrm{C}, 63.64 ; \mathrm{H}, 5.16 ; \mathrm{N}$, 16.34

1H-Benzotriazol-1-yl 4-Amino-5-chloro-2-methoxybenzoate (2c) FAB-MS (positive) $m / z: 319(\mathrm{M}+\mathrm{H})^{+}$. IR $(\mathrm{KBr}) \mathrm{cm}^{-1}: 3422,3322,1786$, 1636. ${ }^{1} \mathrm{H}-\mathrm{NMR}\left(\mathrm{DMSO}-d_{6}\right) \delta: 3.83\left(3 \mathrm{H}, \mathrm{s}, \mathrm{OCH}_{3}\right), 6.46(1 \mathrm{H}, \mathrm{s}, \mathrm{Ar} \mathrm{H}-3)$, $6.58\left(2 \mathrm{H}\right.$, br s, $\left.\mathrm{NH}_{2}\right), 7.51,7.64$ (each $\left.1 \mathrm{H}, \mathrm{dd}, J=7.0,1.0 \mathrm{~Hz}, \mathrm{Bt} \mathrm{Ar}-\mathrm{H}\right), 7.78$ $(1 \mathrm{H}, \mathrm{d}, J=8.0 \mathrm{~Hz}, \mathrm{Bt} \mathrm{Ar}-\mathrm{H}), 8.03(1 \mathrm{H}, \mathrm{s}, \mathrm{Ar} \mathrm{H}-6), 8.14(1 \mathrm{H}, \mathrm{d}, J=8.0 \mathrm{~Hz}, \mathrm{Bt}$ Ar-H). ${ }^{13} \mathrm{C}-\mathrm{NMR}\left(\mathrm{DMSO}-d_{6}\right) \delta: 55.9\left(\mathrm{OCH}_{3}\right), 97.1(\mathrm{Ar} \mathrm{C}-3), 99.0(\mathrm{Ar} \mathrm{C}-1)$, 108.8 (Ar C-5), 109.8 (Bt Ar-C), 119.7 (Bt Ar-C), 125.0 (Bt Ar-C), 127.7 (Bt Ar-C), 128.6 (Bt Ar-C), 132.6 (Ar C-6), 142.7 (Bt Ar-C), 152.6 (Ar C4), 159.4 (Ar C-2), 161.6 (-COO). Anal. Calcd for $\mathrm{C}_{14} \mathrm{H}_{11} \mathrm{~N}_{4} \mathrm{O}_{3} \mathrm{Cl}$ : C, 52.76; H, 3.48; N, 17.58. Found: C, 52.80; H, 3.60; N, 17.63 .

1H-Benzotriazol-1-yl Benzoate (2d) FAB-MS (positive) $\mathrm{m} / \mathrm{z}: 239$ $(\mathrm{M}+\mathrm{H})^{+}$. IR $(\mathrm{KBr}) \mathrm{cm}^{-1}: 1809,1781 .{ }^{1} \mathrm{H}-\mathrm{NMR}\left(\mathrm{CDCl}_{3}\right) \delta: 726-7.28(2 \mathrm{H}$, $\mathrm{m}, \mathrm{Bt} \mathrm{Ar} \mathrm{H}), 7.53-7.57(1 \mathrm{H}, \mathrm{m}, \mathrm{Bt} \mathrm{Ar} \mathrm{H}), 7.59-7.63(2 \mathrm{H}, \mathrm{m}, \mathrm{Ar} \mathrm{H}-3, \mathrm{H}-$ 5), 7.76-7.79 (1H, m, Ar H-4), 8.09-8.11 (1H, m, Bt Ar), 8.27-8.30 $(2 \mathrm{H}, \mathrm{m}, \mathrm{Ar} \mathrm{H}-2, \mathrm{H}-6) \cdot{ }^{13} \mathrm{C}-\mathrm{NMR}\left(\mathrm{CDCl}_{3}\right) \delta: 108.3(\mathrm{Bt} \mathrm{Ar}-\mathrm{C}), 120.6(\mathrm{Bt} \mathrm{Ar}-$ C), 124.8 (Bt Ar-C), 124.9 ( $\mathrm{Ar} \mathrm{C}-1$ ), 128.7 (Bt Ar-C), 128.9 (Bt Ar-C), 129.2 (Ar C-3, C-5), 130.7 (Ar C-2, C-6), 135.5 (Ar C-4), 143.6 (Bt Ar-C), 162.8 (-COO). Anal. Calcd for $\mathrm{C}_{13} \mathrm{H}_{9} \mathrm{~N}_{3} \mathrm{O}_{2}: \mathrm{C}, 65.27 ; \mathrm{H}, 3.79 ; \mathrm{N}, 17.56$. Found: C, 65.47; H, 3.98; N, 17.64

Pyrrolidine-2,5-dion-1-yl Benzoate (2e) FAB-MS (positive) $m / z: 220$
$(\mathrm{M}+\mathrm{H})^{+}$. IR $(\mathrm{KBr}) \mathrm{cm}^{-1}: 1794,1768,1732 .{ }^{1} \mathrm{H}-\mathrm{NMR}\left(\mathrm{CDCl}_{3}\right) \delta: 2.89(4 \mathrm{H}$, s, Pyr), 7.50-7.53 (2H, m, Ar H-3, H-5), 7.66-7.69 (1H, m, Ar H-4), $8.13-8.15(2 \mathrm{H}, \mathrm{m}, \mathrm{Ar} \mathrm{H}-2, \mathrm{H}-6) .{ }^{13} \mathrm{C}-\mathrm{NMR}\left(\mathrm{CDCl}_{3}\right) \delta: 25.7$ (Pyr C-3, C4), 125.2 (Ar C-1), 128.8 (Ar C-3, C-5), 130.5 (Ar C-2, C-6), 134.9 (Ar C4), 161.9 (- $\underline{\mathrm{COO}}$ ), 169.1 (Pyr C-2, C-5). Anal. Calcd for $\mathrm{C}_{11} \mathrm{H}_{9} \mathrm{NO}_{4}$ : C, $60.27 ; \mathrm{H}, 4.14 ; \mathrm{N}, 6.39$. Found: C, 60.24; H, 4.24; N, 6.34 .

Pyrrolidine-2,5-dion-1-yl 3,4-Dichlorophenylacetate (2f) FAB-MS (positive) $m / z: 302(\mathrm{M}+\mathrm{H})^{+}$. IR $(\mathrm{KBr}) \mathrm{cm}^{-1}: 1821,1784,1730 .{ }^{1} \mathrm{H}-\mathrm{NMR}$ $\left(\mathrm{CDCl}_{3}\right) \delta: 2.83(4 \mathrm{H}, \mathrm{s}, \mathrm{Pyr}), 3.89\left(2 \mathrm{H}, \mathrm{s}, \mathrm{CH}_{2} \mathrm{Ph}\right), 7.18-7.19(1 \mathrm{H}, \mathrm{m}, \mathrm{Ar}$ $\mathrm{H}-6), 7.43-7.46(2 \mathrm{H}, \mathrm{m}, \mathrm{Ar} \mathrm{H}-2, \mathrm{H}-5) .{ }^{13} \mathrm{C}-\mathrm{NMR}\left(\mathrm{CDCl}_{3}\right) \delta: 25.6$ (Pyr C3, C-4), 36.7 ( $\left.\mathrm{CH}_{2} \mathrm{Ph}\right), 128.6$ (Ar C-6), 130.8 (Ar C-5), 131.3 (Ar C-2), 131.4 (Ar C-4), 132.3 (Ar C-3), 132.9 (Ar C-1), 165.9 (- - OO), 168.7 (Pyr C-2, C-5). Anal. Calcd for $\mathrm{C}_{12} \mathrm{H}_{9} \mathrm{NO}_{4} \mathrm{Cl}_{2}: \mathrm{C}, 47.71 ; \mathrm{H}, 3.00 ; \mathrm{N}, 4.64$. Found: C, 47.75; H, 3.10; N, 4.66.

Preparation of 2-[(2-Benzoylcyclohexane)carboxamido]-3-(piperidin1-yl)propanoic Acid (4a) A solution of $\beta$-aminoalanine dihydrochloride (3a) $(0.35 \mathrm{~g}, 1.43 \mathrm{mmol})$ in potassium bicarbonate $\left(\mathrm{K}_{2} \mathrm{CO}_{3}\right)(0.99 \mathrm{~g}$, $7.17 \mathrm{mmol})$ in $\mathrm{H}_{2} \mathrm{O}(\mathrm{ca} .8 \mathrm{ml})$ was added to a solution of activated carboxylic acid (2a) $(0.5 \mathrm{~g}, 1.43 \mathrm{mmol})$ in $\mathrm{CH}_{2} \mathrm{Cl}_{2}$, then $\mathrm{EtOH}(10 \mathrm{ml})$ was added to this solution. After stirring for $5 \mathrm{~h}$ at room temperature, the reaction mixture was concentrated under reduced pressure and the residue triturated with EtOH to give a solid material. After separation of this product by filtration, the filtrate was concentrated, and purification of the product by silica gel column with ethanol as solvent afford 4a $(0.49 \mathrm{~g}, 89 \%)$. FAB-MS (positive) $\mathrm{m} / \mathrm{z}: 387$ $(\mathrm{M}+\mathrm{H})^{+}$. IR $(\mathrm{KBr}) \mathrm{cm}^{-1}: 3363,3262,1665,1640,1607 .{ }^{1} \mathrm{H}-\mathrm{NMR}\left(\mathrm{CDCl}_{3}\right)$ $\delta: 1.25-2.12(14 \mathrm{H}, \mathrm{m}, \mathrm{Ppd} \mathrm{H}-3, \mathrm{H}-4, \mathrm{H}-5$ and Cyhx H-3, H-4, H-5, H-6), $2.72-2.87(1 \mathrm{H}, \mathrm{m}, \mathrm{Cyhx} \mathrm{H}-1), 2.92-3.35\left(6 \mathrm{H}, \mathrm{m}, \mathrm{Ppd} \mathrm{H} 2, \mathrm{H}-6\right.$ and $\mathrm{CH}_{2}-$ 1-piperidinyl), 3.62-3.67 (1H, m, Cyhx H-6), 4.26- 4.30 (1H, m, NHC$\mathrm{COOH}), 5.0-7.0(1 \mathrm{H}, \mathrm{br}, \mathrm{COOH}), 7.47(1 \mathrm{H}, \mathrm{dd}, J=15.0,7.5 \mathrm{~Hz}, \mathrm{CONH})$, $7.50-7.56(3 \mathrm{H}, \mathrm{m}, m$, and $p-\mathrm{Ar}-\mathrm{H}), 7.96-7.98(2 \mathrm{H}, \mathrm{m}, o-\mathrm{Ar}-\mathrm{H}) .{ }^{13} \mathrm{C}-\mathrm{NMR}$ $\left(\mathrm{CDCl}_{3}\right) \delta: 22.1,22.2,22.8,25.5,25.6,29.8,30.0$ (Cyhx C-3-C-6 and Ppd C-3-C-5), 46.2 (Cyhx C-1), 46.9 (Cyhx C-2), 49.2 (CONHCHCOOH), $53.9(\times 2)\left(\right.$ Ppd C-2, C-6), $56.9\left(\mathrm{CH}_{2}-1\right.$-piperidinyl $), 128.5(\times 3), 128.6(\mathrm{Ar}$ C-2, C-3, C-5, C-6), 132.7 (Ar C-4), 136.5 (Ar C-1), 172.3 ( $\mathrm{COOH}), 175.4$ $(\underline{\mathrm{CONH}}), 203.2(\underline{\mathrm{COPh}})$. Anal. Calcd for $\mathrm{C}_{22} \mathrm{H}_{30} \mathrm{~N}_{2} \mathrm{O}_{4} \cdot \mathrm{H}_{2} \mathrm{O}: \mathrm{C}, 65.32 ; \mathrm{H}$, 7.97; N, 6.93. Found: C, 65.25; H, 8.00; N, 6.92.

Preparation of 2-[3-Fluoro-4-(piperidin-1-yl)benzamido]-3-(piperidin-1-yl)propanoic Acid (4b) A solution of $\beta$-aminoalanine dihydrochloride (3a) $(0.43 \mathrm{~g}, 1.76 \mathrm{mmol})$ and $\mathrm{K}_{2} \mathrm{CO}_{3}(1.21 \mathrm{~g}, 8.76 \mathrm{mmol})$ in $\mathrm{H}_{2} \mathrm{O}$ (ca. $10 \mathrm{ml})$ was added to a solution of activated carboxylic acid (2b) $(0.6 \mathrm{~g}$, $1.76 \mathrm{mmol})$ in acetonitrile $\left(\mathrm{CH}_{3} \mathrm{CN}\right)$, and the mixture was stirred for $0.5 \mathrm{~h}$. After concentration under reduced pressure, $1 \mathrm{~N}$-hydrochloric acid $(1 \mathrm{~N}-\mathrm{HCl})$ was added to make the solution $\mathrm{pH} c a$. 5. Evaporation of the solvent gave a viscous residue, and then trituration of this product with EtOH afforded solid material. After separation of this material by filtration, the filtrate was concentrated and purified through a silica gel column (ethanol $\rightarrow$ ethanol/ammonia as solvent) to give $4 \mathbf{b}(0.52 \mathrm{~g}, 78 \%$ ). FAB-MS (positive) $\mathrm{m} / \mathrm{z}: 378$ $(\mathrm{M}+\mathrm{H})^{+}$. IR $(\mathrm{KBr}) \mathrm{cm}^{-1}: 3390,3260,1650,1617 .{ }^{1} \mathrm{H}-\mathrm{NMR}$ (DMSO- $\left.d_{6}\right) \delta$ : $1.43-1.46(2 \mathrm{H}, \mathrm{m}, \mathrm{Ppd} \mathrm{H}-4), 155-1.67$ [10H, m, (Ppd H-3 $\times 2, \mathrm{H}-5 \times 2$ and $\mathrm{H}-4) \times 2], \quad 2.69-2.94\left[10 \mathrm{H}, \mathrm{m},(\mathrm{Ppd} \mathrm{H}-2 \times 2, \mathrm{H}-6 \times 2) \times 2\right.$ and $\mathrm{C}_{2}-1-$ piperidinyl], $4.50-4.51(1 \mathrm{H}, \mathrm{m}, \mathrm{NHCHCOOH}), 4.65(1 \mathrm{H}, \mathrm{br}, \mathrm{COOH}), 7.05$ $(1 \mathrm{H}, \mathrm{t}, J=8.5 \mathrm{~Hz}, \mathrm{Ar} \mathrm{H}-2), 7.58-7.63(2 \mathrm{H}, \mathrm{m}, \mathrm{Ar} \mathrm{H}-5, \mathrm{H}-6), 8.24(1 \mathrm{H}, \mathrm{d}$, $J=7.0 \mathrm{~Hz}, \mathrm{NH}) .{ }^{13} \mathrm{C}-\mathrm{NMR}\left(\mathrm{DMSO}-d_{6}\right) \delta: 22.7,23.6(\mathrm{Ppd} \mathrm{C}-4), 24.5(\times 2)$, $25.4(\times 2)($ Ppd C-3, C-5), $48.6(\underline{\mathrm{CHCOOH}}), 51.8(\times 4)(\mathrm{Ppd} \mathrm{C}-2, \mathrm{C}-6), 64.8$ ( $\mathrm{CH}_{2}$-1-piperidinyl), 114.8, (Ar C-5), 118.3 (Ar C-2), 124.0 (Ar C-6), 126.7 (Ar C-1), 143.0 (Ar C-4), 153.7 (d, $J=244.1 \mathrm{~Hz}, \mathrm{Ar} \mathrm{C}-3$ ), 164.5 (ㅇNH), $172.4(\mathrm{COOH})$. Anal. Calcd for $\mathrm{C}_{20} \mathrm{H}_{28} \mathrm{~N}_{3} \mathrm{O}_{3} \mathrm{~F} \cdot 0.3 \mathrm{H}_{2} \mathrm{O}: \mathrm{C}, 62.74 ; \mathrm{H}, 7.53 ; \mathrm{N}$, 10.98. Found: C, 62.73; H, 7.70; N, 10.75 .

Preparation of 2-(4-Amino-5-chloro-2-methoxy)benzamido-3-(piperidin-1-yl)propanoic Acid (4c) Reaction of activated carboxylic acid (2c) $(0.5 \mathrm{~g}, 1.57 \mathrm{mmol})$ with $\beta$-aminoalanine dihydrochloride (3a) $(0.39 \mathrm{~g}$, $1.59 \mathrm{mmol})$ was carried out in the same manner described above in the preparation of $\mathbf{4 b}$. The isolated crude product was purified by column chromatography $\left(\mathrm{SiO}_{2} /\right.$ ethanol) to give (4c) $(0.48 \mathrm{~g}, 86 \%)$. FAB-MS (positive) $m / z: 356(\mathrm{M}+\mathrm{H})^{+}$. IR $(\mathrm{KBr}) \mathrm{cm}^{-1}: 3460,3368,3206,1680,1624,1590 .{ }^{1} \mathrm{H}-$ NMR (DMSO- $\left.d_{6}\right) \delta: 1.48(2 \mathrm{H}, \mathrm{t}, J=5.5 \mathrm{~Hz}, \mathrm{Ppd} \mathrm{H}-4), 1.62-1.63(4 \mathrm{H}, \mathrm{m}$, $\mathrm{H}-3$, Ppd H-5), 2.80-2.84 (3H, m, Ppd H-2, H-6 and $\mathrm{C}_{\mathrm{A}} \mathrm{H}_{\mathrm{B}}-1-$ piperidinyl), 2.91-2.94 (2H, m, Ppd H-2, H-6), 3.15 (1H, dd, $J=12.0$, $6.0 \mathrm{~Hz}, \mathrm{CH}_{\mathrm{A}} \underline{\mathrm{H}}_{\mathrm{B}}$-1-piperidinyl $), 3.86\left(3 \mathrm{H}, \mathrm{s}, \mathrm{OCH}_{3}\right), 4.0-5.6(1 \mathrm{H}, \mathrm{br}$, $\mathrm{COOH}), 4.30-4.32(1 \mathrm{H}, \mathrm{m}, \mathrm{NHC} \underline{\mathrm{HCOOH}}), 5.97\left(2 \mathrm{H}, \mathrm{s}, \mathrm{NH}_{2}\right), 6.52(1 \mathrm{H}, \mathrm{s}$, Ar H-3), $7.73(1 \mathrm{H}, \mathrm{s}, \mathrm{Ar} \mathrm{H}-6), 8.58(1 \mathrm{H}, \mathrm{d}, J=5.0 \mathrm{~Hz}, \mathrm{NH}) .{ }^{13} \mathrm{C}-\mathrm{NMR}$ $\left(\mathrm{DMSO}-d_{6}\right) \delta$ : 22.3 (Ppd C-4), 24.2 (Ppd C-3, C-5), 48.7 (NHCHCOOH), 52.6 (Ppd C-2, C-6), $56.0\left(\mathrm{OCH}_{3}\right), 57.3\left(\mathrm{CH}_{2}-1\right.$-piperidinyl), 97.6 (Ar C-3), 109.0 (Ar C-1), 109.6 (Ar C-5), 131.5 (Ar C-6), 148.7 (Ar C-4), 157.7 (Ar 
C-2), $163.3(\underline{\mathrm{CONH}}), 171.9(\underline{\mathrm{COOH}})$. Anal. Calcd for $\mathrm{C}_{16} \mathrm{H}_{22} \mathrm{~N}_{3} \mathrm{O}_{4} \mathrm{Cl}$ 1.3 $\mathrm{H}_{2} \mathrm{O}: \mathrm{C}, 50.67 ; \mathrm{H}, 6.54 ; \mathrm{N}, 11.08$. Found: C, 50.80; H, 6.36; N, 11.01 .

Preparation of 2-Benzamido-3-(dimethylamino)propanoic Acid (4d) A solution of $\beta$-aminoalanine dihydrochloride (3b) $(0.239 \mathrm{~g}, 1.0 \mathrm{mmol})$ and $\mathrm{K}_{2} \mathrm{CO}_{3}(0.691 \mathrm{~g}, 5.0 \mathrm{mmol})$ in $\mathrm{H}_{2} \mathrm{O}(\mathrm{ca} .4 \mathrm{ml})$ was added to the solution of activated carboxylic acid $(\mathbf{2 d})(0.239 \mathrm{~g}, 1.0 \mathrm{mmol})$ in $\mathrm{CH}_{2} \mathrm{Cl}_{2}$. After stirring for $5 \mathrm{~h}$ at $30^{\circ} \mathrm{C}$, the reaction mixture was concentrated under reduced pressure and the residue triturated with EtOH to give a solid material. After separation of this product by filtration, the filtrate was concentrated and eluted by a silica gel column with methanol as solvent. The collected product was neutralized by addition of $\mathrm{HCl} / \mathrm{EtOH}(c a \cdot \mathrm{pH}=7)$ and dried under reduced pressure. The residue was dissolved into $\mathrm{EtOH}$ and insoluble material removed by filtration, and evaporation of the filtrate gave $\mathbf{4 d}(0.18 \mathrm{~g}, 76 \%)$. Analytical sample was obtained by recrystallization from EtOH. FAB-MS (positive) $\mathrm{m} / z: 237(\mathrm{M}+\mathrm{H})^{+}$. IR $(\mathrm{KBr}) \mathrm{cm}^{-1}: 3395,1653,1620 .{ }^{1} \mathrm{H}-\mathrm{NMR}$ $\left(\mathrm{D}_{2} \mathrm{O}\right) \delta: 3.01\left(6 \mathrm{H}, \mathrm{s}, \mathrm{CH}_{3} \times 2\right), 3.50\left(1 \mathrm{H}, \mathrm{dd}, J=13.0,8.0 \mathrm{~Hz}, \mathrm{C}_{\mathrm{A}} \mathrm{H}_{\mathrm{B}}-\mathrm{N}=\right)$, $3.65\left(1 \mathrm{H}, \mathrm{dd}, J=13.0,6.5 \mathrm{~Hz}, \mathrm{CH}_{\mathrm{A}} \underline{\mathrm{H}}_{\mathrm{B}}-\mathrm{N}=\right), 4.83(1 \mathrm{H}, \mathrm{dd}, J=8.0,6.5 \mathrm{~Hz}$, $\mathrm{NHCHCOOH}), 7.57(2 \mathrm{H}, \mathrm{t}, J=8.0 \mathrm{~Hz}, m-\mathrm{Ar}-\mathrm{H}), 7.66(1 \mathrm{H}, \mathrm{t}, J=7.0 \mathrm{~Hz}, p-$ Ar-H). 7.86-7.88 (2H, m, o-Ar-H). ${ }^{13} \mathrm{C}-\mathrm{NMR}\left(\mathrm{D}_{2} \mathrm{O}\right) \delta: 46.2\left(\mathrm{CH}_{3} \times 2\right), 53.0$ $(\mathrm{CONH} \underline{\mathrm{CHCOOH}}), 61.7\left(\underline{\mathrm{CH}}_{2} \mathrm{~N}=\right), 130.2(\mathrm{Ar} \mathrm{C}-3, \mathrm{C}-5), 131.7(\mathrm{Ar} \mathrm{C}-2, \mathrm{C}-$ 6), 135.4 (Ar C-4), 135.6 (Ar C-1), 173.4 ( $\mathrm{CONH}), 176.8$ (COOH). Anal. Calcd for $\mathrm{C}_{12} \mathrm{H}_{16} \mathrm{~N}_{2} \mathrm{O}_{3}$ : C, 61.00; H, 6.83; N, 11.86. Found: C, 60.92; H, 6.86; N,11.89.

Preparation of 2-(4-Amino-5-chloro-2-methoxy)benzamidopropanoic Acid (4e) A solution of DL-alanine $(0.20 \mathrm{~g}, 2.25 \mathrm{mmol})$ and $\mathrm{K}_{2} \mathrm{CO}_{3}(0.95 \mathrm{~g}$, $6.88 \mathrm{mmol})$ in $\mathrm{H}_{2} \mathrm{O}(\mathrm{ca} .7 \mathrm{ml})$ was added to a solution of activated carboxylic acid $(\mathbf{2 c})(0.72 \mathrm{~g}, 2.26 \mathrm{mmol})$ in DMF, then the mixture was stirred for $10 \mathrm{~min}$ at room temperature. After concentration of the solvent, the residue was acidified with $1 \mathrm{~N}-\mathrm{HCl}$ and the precipitated material collected by filtration. The precipitated materials were washed with AcOEt $(100 \mathrm{ml})$ to give a high purity of $4 \mathrm{e}(0.55 \mathrm{~g}, 90 \%)$. Analytical sample was obtained by recrystallization from acetonitrile. FAB-MS (positive) $\mathrm{m} / \mathrm{z}: 273(\mathrm{M}+\mathrm{H})^{+}$. IR $(\mathrm{KBr}) \mathrm{cm}^{-1}: 3472,3391,3320,3196,1719,1624 .{ }^{1} \mathrm{H}-\mathrm{NMR}$ (DMSO- $d_{6}$ ) $\delta: 1.37\left(3 \mathrm{H}, \mathrm{d}, J=7.0 \mathrm{~Hz}, \mathrm{CH}_{3}\right), 2.50\left(3 \mathrm{H}, \mathrm{s}, \mathrm{OCH}_{3}\right), 4.38-4.44(1 \mathrm{H}, \mathrm{m}$, CONHCH), $5.95\left(2 \mathrm{H}, \mathrm{s}, \mathrm{NH}_{2}\right), 6.51(1 \mathrm{H}, \mathrm{s}, \mathrm{Ar} \mathrm{H}-3), 7.71(1 \mathrm{H}, \mathrm{s}, \mathrm{Ar} \mathrm{H}-6)$ $8.19(1 \mathrm{H}, \mathrm{s}, \mathrm{CONH}), 12.5(1 \mathrm{H}, \mathrm{br}, \mathrm{COOH}) .{ }^{13} \mathrm{C}-\mathrm{NMR}$ (DMSO- $\left.d_{6}\right) \delta: 17.9$ $\left(\mathrm{CH}_{3}\right), 47.9(\mathrm{NHCHCOOH}), 56.0\left(\mathrm{OCH}_{3}\right), 97.6(\mathrm{Ar} \mathrm{C}-3), 109.1(\mathrm{Ar} \mathrm{C}-1)$ 109.7 (Ar C-5), 131.5 (Ar C-6), 148.7 (Ar C-4), 157.5 (Ar C-2), 163.0 ( $\underline{\mathrm{CONH}}), 174.1(\underline{\mathrm{COOH}})$. Anal. Calcd for $\mathrm{C}_{11} \mathrm{H}_{13} \mathrm{~N}_{2} \mathrm{O}_{4} \mathrm{Cl}$ : C, 48.45; H, 4.81; $\mathrm{N}, 10.27$. Found: $\mathrm{C}, 48.55 ; \mathrm{H}, 4.84 ; \mathrm{N}, 10.49$.

Compound (4e) was also obtained from the reaction of $2 \mathrm{~g}^{13)}$ with DL-alanine. Thus a solution of DL-alanine $(0.20 \mathrm{~g}, 2.25 \mathrm{mmol})$ and $\mathrm{K}_{2} \mathrm{CO}_{3}(0.95 \mathrm{~g}$, $6.88 \mathrm{mmol})$ in $\mathrm{H}_{2} \mathrm{O}(8 \mathrm{ml})$ was added to a solution of activated carboxylic acid $(2 \mathrm{~g})(0.67 \mathrm{~g}, 2.25 \mathrm{mmol})$ in DMF, EtOH $(10 \mathrm{ml})$ was added to the solution, then the mixture was stirred for $0.5 \mathrm{~h}$ at room temperature. After concentration of the solvent, the residue was acidified with $1 \mathrm{~N}-\mathrm{HCl}$ and the precipitated material collected by filtration to give $4 \mathrm{e}(0.56 \mathrm{~g}, 92 \%)$.

Preparation of 2-Benzamido-3-phenylpropanoic Acid (4f) A solution of DL-phenylalanine $(0.38 \mathrm{~g}, 2.30 \mathrm{mmol})$ and $\mathrm{K}_{2} \mathrm{CO}_{3}(0.95 \mathrm{~g}, 6.88 \mathrm{mmol})$ in $\mathrm{H}_{2} \mathrm{O}(\mathrm{ca} .7 \mathrm{ml})$ was added to the solution of activated carboxylic acid (2e) $(0.5 \mathrm{~g}, 2.28 \mathrm{mmol})$ in $\mathrm{CH}_{2} \mathrm{Cl}_{2}$ and stirred for $2 \mathrm{~h}$. After removal of the solvent under reduced pressure, the residue was acidified with $1 \mathrm{~N}-\mathrm{HCl}$. The precipitate was extracted with AcOEt, washed with brine, then dried over $\mathrm{Na}_{2} \mathrm{SO}_{4}$. Evaporation of the solvent gave a high purity of $\mathbf{4 f}(0.6 \mathrm{~g}, 97 \%)$. Analytical sample was obtained from recrystallization from acetic acid. FAB-MS (positive) $m / z: 270(\mathrm{M}+\mathrm{H})^{+}$. IR $(\mathrm{KBr}) \mathrm{cm}^{-1}: 3328,1721,1613 .{ }^{1} \mathrm{H}-\mathrm{NMR}$ $\left(\mathrm{CDCl}_{3}\right) \delta: 3.24-3.27\left(1 \mathrm{H}, \mathrm{m}, \mathrm{CH}_{\mathrm{A}} \mathrm{H}_{\mathrm{B}}-\mathrm{Ph}\right), 3.34-3.38\left(1 \mathrm{H}, \mathrm{m}, \mathrm{CH}_{\mathrm{A}} \underline{\mathrm{H}}_{\mathrm{B}}-\right.$ $\mathrm{Ph}), 5.06-5.10(1 \mathrm{H}, \mathrm{m}, \mathrm{CONHCHCOOH}), 6.60(1 \mathrm{H}, \mathrm{d}, J=7.5 \mathrm{~Hz}, \mathrm{NH})$, $6.95(1 \mathrm{H}, \mathrm{br}, \mathrm{COOH}), 7.19-7.68(10 \mathrm{H}, \mathrm{m}, \mathrm{Ar}-\mathrm{H}) .{ }^{13} \mathrm{C}-\mathrm{NMR}\left(\mathrm{CDCl}_{3}\right) \delta$ : $37.3\left(\mathrm{CH}_{2} \mathrm{Ph}\right), 53.7(\mathrm{NH} \underline{\mathrm{C} H C O O H}), 127.1,127.3,128.7(\times 2), 129.4,132.0$ (Ar C2-C5), 133.5, 135.6, (Ar-C1), 167.8 (CONH), 174.8 (COOH). Anal. Calcd for $\mathrm{C}_{16} \mathrm{H}_{15} \mathrm{NO}_{3} \cdot 0.1 \mathrm{H}_{2} \mathrm{O}: \mathrm{C}, 70.89 ; \mathrm{H}, 5.65 ; \mathrm{N}, 5.17$. Found: $\mathrm{C}, 70.85$; $\mathrm{H}, 5.74 ; \mathrm{N}, 5.06$.

Preparation of 2-[2-(3,4-Dichlorophenyl)acetamido]propanoic Acid (4g) A solution of DL-alanine $(0.147 \mathrm{~g}, 1.65 \mathrm{mmol})$ and $\mathrm{K}_{2} \mathrm{CO}_{3}(0.69 \mathrm{~g}$, $5.00 \mathrm{mmol})$ in $\mathrm{H}_{2} \mathrm{O}(\mathrm{ca} .6 \mathrm{ml})$ was added to the solution of activated carboxylic acid (2f) $(0.50 \mathrm{~g}, 1.66 \mathrm{mmol})$ in DMF and stirred for $10 \mathrm{~min}$ at room temperature. Precipitated material by addition of water was collected by filtration to afford $\mathbf{4 g}(0.40 \mathrm{~g}, 89 \%)$. Analytical sample was obtained by recrystallization from $\mathrm{CH}_{3} \mathrm{CN}$. FAB-MS (positive) $m / z: 276(\mathrm{M}+\mathrm{H})^{+}$. IR $(\mathrm{KBr})$ $\mathrm{cm}^{-1}: 3295,1748,1612 .{ }^{1} \mathrm{H}-\mathrm{NMR}$ (DMSO- $\left.d_{6}\right) \delta: 1.27\left(3 \mathrm{H}, \mathrm{s}, \mathrm{CH}_{3}\right), 3.17$ $(1 \mathrm{H}, \mathrm{br}, \mathrm{COOH}), 3.49\left(2 \mathrm{H}, \mathrm{s}, \mathrm{C}_{2} \mathrm{Ph}\right), 4.20(1 \mathrm{H}, \mathrm{t}, J=7.3 \mathrm{~Hz}, \mathrm{CONHC} \underline{\mathrm{H}})$, $7.24-7.26(1 \mathrm{H}, \mathrm{m}, \mathrm{Ar}-\mathrm{H}), 7.53-7.55(2 \mathrm{H}, \mathrm{m}, \mathrm{Ar}-\mathrm{H}), 8.40(1 \mathrm{H}, \mathrm{d}$, $J=7.3 \mathrm{~Hz}, \mathrm{NH}) .{ }^{13} \mathrm{C}-\mathrm{NMR}$ (DMSO- $\left.d_{6}\right) \delta: 17.1\left(\mathrm{CH}_{3}\right), 40.5\left(\underline{\mathrm{CH}}_{2} \mathrm{Ph}\right), 47.6$ (CONHㄷCOOOH), 129.0 129.4, 130.1, 130.5, 130.9 (Ar-C), 137.3 (Ar C1), $168.9(\underline{\mathrm{CONH}}), 173.9(\underline{\mathrm{COOH}})$. Anal. Calcd for $\mathrm{C}_{11} \mathrm{H}_{11} \mathrm{NO}_{3} \mathrm{Cl}_{2}: \mathrm{C}, 47.85$; H, 4.02; N, 5.07. Found: C, 47.98; H, 4.03; N, 5.10.

One-Pot Synthesis of $4 \mathrm{f}$ from 1d. (Method A) A combination of consecutive steps that saves time and makes the procedure conventional was devised. The procedure can be depicted as follows: To a stirred solution of carboxylic acid (1d) $(0.5 \mathrm{~g}, 4.10 \mathrm{mmol})$ in $\mathrm{CH}_{2} \mathrm{Cl}_{2}(5 \mathrm{ml})$ was added $\mathrm{HO}-\mathrm{Su}$ $(0.52 \mathrm{~g}, 4.51 \mathrm{mmol})$ and WSCI $(0.86 \mathrm{~g}, 4.51 \mathrm{mmol})$ at $35^{\circ} \mathrm{C}$ and the resulting mixture was stirred for $10 \mathrm{~min}$. To this solution was added a solution of DLphenylalanine $(0.677 \mathrm{~g}, 4.10 \mathrm{~mol})$ and $\mathrm{K}_{2} \mathrm{CO}_{3}(3.40 \mathrm{~g}, 24.6 \mathrm{mmol})$ in $\mathrm{H}_{2} \mathrm{O}$ (ca. $25 \mathrm{ml}$ ) then the mixture was kept stirred at $35^{\circ} \mathrm{C}$ for $3 \mathrm{~h}$. After removal of the solvent under reduced pressure, the residue was acidified with $1 \mathrm{~N}$ $\mathrm{HCl}$. The precipitate was extracted with AcOEt, washed with water and brine, then dried over $\mathrm{Na}_{2} \mathrm{SO}_{4}$. Evaporation of the solvent gave (4f) $(1.05 \mathrm{~g}$, $95 \%)$.

(Method B) To a stirred solution of carboxylic acid (1d) $(0.16 \mathrm{~g}$, $1.33 \mathrm{mmol})$ in $\mathrm{CH}_{2} \mathrm{Cl}_{2}(3 \mathrm{ml})$ was added $\mathrm{HO}-\mathrm{Bt}(0.27 \mathrm{~g}, 1.74 \mathrm{mmol})$ and WSCI $(0.33 \mathrm{~g}, 1.74 \mathrm{mmol})$ at $35^{\circ} \mathrm{C}$ and the resulting mixture was stirred for $10 \mathrm{~min}$. To this solution was added a solution of DL-phenylalanine $(0.22 \mathrm{~g}$, $1.33 \mathrm{mmol})$ and $\mathrm{K}_{2} \mathrm{CO}_{3}(1.10 \mathrm{~g}, 7.97 \mathrm{mmol})$ in $\mathrm{H}_{2} \mathrm{O}(c a .8 \mathrm{ml})$ then the mixture was kept stirred at $35^{\circ} \mathrm{C}$ for $3 \mathrm{~h}$. After removal of the solvent under reduced pressure, the residue was acidified with concentrated $\mathrm{HCl}$. The precipitate was extracted with $\mathrm{AcOEt}$, washed with concentrated $\mathrm{HCl}$, water, and brine, then dried over $\mathrm{Na}_{2} \mathrm{SO}_{4}$. Evaporation of the solvent gave (4f) $(0.30 \mathrm{~g}$, $84 \%)$

\section{References and Notes}

1) Jursic B. S., Neumann D., Synth. Commun., 31, 555-564 (2001), and related references cited therein.

2) Gross M., Meinhofer J., "The Peptides," Academic Press, New York, 1979 , and related references cited therein.

3) Alberico F., Carpino L. A., "Coupling Reagents and Activation Method in Enzymology," Vol. 289, Academic Press, New York, 1997, p. 104, and related references cited therein.

4) Ottl J., Musiol H. J., Moroder L., J. Peptide Sci., 5, 103-110 (1999).

5) Gagnon P., Huang X., Therrien E., Keillor J. W., Tetraheron Lett., 43, $7717-7719$ (2002).

6) Schmidt U., Utz R., Lieberknecht A., Griesser H., Potzolli B., Bahr J., Wagner K., Fischer P., Synthesis, 1987, 236-241 (1987).

7) Katritzky A. R., Suzuki K., Singh S. K., Synthesis, 2004, 2645-2652 (2004).

8) Fujisaki F., Abe N., Sumoto K., Chem. Pharm. Bull., 50, 129-132 (2002).

9) Fujisaki F., Abe N., Sumoto K., Chem. Pharm. Bull., 52, 1238-1241 (2004).

10) Konig W., Geiger R., Chem. Ber., 103, 788-798 (1970).

11) This one-pot synthesis needed a large excess amount of aq. potassium bicarbonate for neutralization

12) Abe N., Fujisaki F., Sumoto K., Chem. Pharm. Bull., 46, 142-144 (1998), and related references cited therein.

13) This activated intermediate (2g) was obtained in a similar manner as that described for the general procedure of compound (2). Compound (2g); $\mathrm{mp} 238^{\circ} \mathrm{C}$ (dec.) $\left(\mathrm{CH}_{3} \mathrm{CN}\right)$. FAB-MS (positive) $\mathrm{m} / \mathrm{z}: 298$ $(\mathrm{M}+\mathrm{H})^{+}$. IR $(\mathrm{KBr}) \mathrm{cm}^{-1}: 1796,1773,1720 .{ }^{1} \mathrm{H}-\mathrm{NMR}$ (DMSO- $d_{6}$, $500 \mathrm{MHz}) \delta: 2.83(4 \mathrm{H}, \mathrm{s}, \mathrm{Pyr}), 3.78\left(3 \mathrm{H}, \mathrm{s}, \mathrm{OCH}_{3}\right), 6.52(1 \mathrm{H}, \mathrm{s}, \mathrm{Ar} \mathrm{H}-$ 3), $6.60\left(2 \mathrm{H}\right.$, br s, $\left.\mathrm{NH}_{2}\right), 7.73(1 \mathrm{H}, \mathrm{s}, \mathrm{Ar} \mathrm{H}-6) .{ }^{13} \mathrm{C}-\mathrm{NMR}$ (DMSO- $d_{6}$, $125 \mathrm{MHz}) \delta$ : $25.4(\times 2)(\mathrm{Pyr} \mathrm{C}-3, \mathrm{C}-4), 55.8\left(\mathrm{OCH}_{3}\right), 97.3(\mathrm{Ar} \mathrm{C}-3)$, 100.0 (Ar C-1), 108.4 (Ar C-5), 132.6 (Ar C-6), 152.0 (Ar C-4), 158.6 (Ar C-2), $161.0(-\mathrm{COO}), 170.6(\times 2)($ Pyr C-2, C-5). Anal. Calcd for $\mathrm{C}_{12} \mathrm{H}_{11} \mathrm{~N}_{2} \mathrm{O}_{5} \mathrm{Cl}: \mathrm{C}, 48.26 ; \mathrm{H}, 3.71 ; \mathrm{N}, 9.38$. Found: C, 48.25; H, 3.78; $\mathrm{N}, 9.51$.

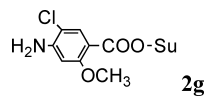

14) Bailén M. A., Chinchilla R., Dodsworth D. J., Nájera C., Tetrahedron Lett., 43, 1661-1664 (2002). 